\title{
Comparison of Efficiency of Different Shapes of Homogeneous and Two-Part Piezo Elements on Vibration Reduction
}

\author{
R. TROJANOWSKI* AND J. WiCIAK \\ Department of Mechanics and Vibroacoustics, AGH - University of Science and Technology \\ al. A. Mickiewicza 30, 30-059 Kraków, Poland
}

The subject of this work is a numerical analysis of efficiency of different piezo element shapes and types used for vibration (and later structural sound) reduction. The shapes of both types of piezo elements used are as follows: square, right-angled triangle, and parallelogram. There are 2 types of piezo elements used in this study: homogeneous elements and elements consisting of 2 parts (inner and outer) of different composition. These are attached to steel plate clamped on all sides. For every simulation 5 elements are used as actuators, with 1 as disturbance source, and other 4 as active dampers. On the other side of a plate 4 elements are placed acting as vibration sensors. The actuator used as a disturbance source as well as sensors are always in the shape of a square. 4 modes were taken into account (analyzed each one singular and all together). Analyses were made with the use of ANSYS software.

PACS: 43.28.Js, 43.40.-r, 46.40.Ff

\section{Preface}

For most machines vibrations have a detrimental effect on their performance and "lifespan". For humans prolonged exposure to excessive vibration also has negative health effects. Therefore much effort is made to lower (or possibly eliminate) unwanted vibrations. One of the approach to this problem is by the use of so-called active methods of vibration control $[1,2]$. In those active control methods there is a group that uses piezo elements. These elements first introduced in works of Fuller, Dimitradis [3], are constantly improving. Better materials allow for higher force output from applied voltage. Many works concerning piezo elements deal with issues like: control type and algorithms [4-7], optimal element placement [8-11], combining them with passive solutions to improve transmission loss [12], or using them for noise control [13-15]. There are almost no works dealing with how shape of piezo actuator affects its efficiency, or if using some other type than homogeneous element could be beneficial.

\section{Description}

The subject of this work was a numerical analysis of the effect that different shapes and types of piezo elements have on vibration reduction. For this purpose numerical models had been constructed. Those models consisted of

\footnotetext{
* corresponding author; e-mail:

roman.cz.trojanowski@agh.edu.pl
}

steel plate clamped on all sides with a number of piezo elements attached to it (on one side 4 acting as sensors, and on the other 5 acting as actuators). 1 of the actuators and all sensors were always square shape homogeneous piezo elements (as it was assumed that plate excitation and vibration detection should be the same for all analyses). The 4 other actuators had 3 different shapes (square, parallelogram and right-angled triangle) and 2 different make-ups (either homogeneous composition or consisting of 2 parts inner and outer with inner part having dimensions twice smaller than the outer part) with the restriction that the total area of the piezoelement is constant (and equals $1600 \mathrm{~mm}^{2}$ ). It should be noted that the two-part types of actuators had voltage applied only to the outer part of piezoelement.

This amounted to 6 models total. For each numerical model analyses were performed for 5 first mode shapes. Although it should be noted because modes $(1,2)$ and $(2,1)$ have the same (or almost the same) frequency, 4 analyses were performed for singular modes and additional analysis for all modes. Figure 1 shows model dimensions and piezo elements placement.

The actuators number and distribution result from an attempt to reduce possibly large number of plate modes.

The voltage applied to piezoelements was given as

$$
V_{i}=V_{\mathrm{a} i} \mathrm{e}^{\mathrm{j}\left(\omega t+\varphi_{i}\right)},
$$

where $V_{i}$ - voltage applied to piezoceramic, $V_{\mathrm{a} i}$ - amplitude of voltage applied to piezoceramic, $\varphi_{i}$ - phase angle of voltage applied to piezoceramic.

The parameter values used in model are shown in Table I. 


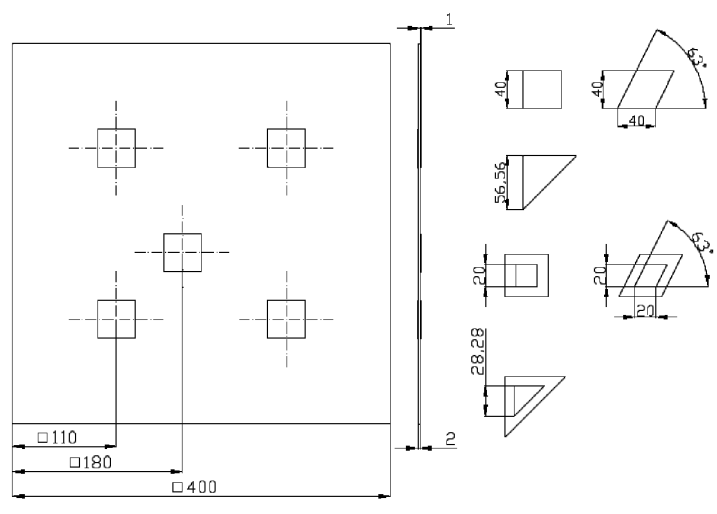

Fig. 1. Model dimensions and piezoelements placement.

Model parameters.

TABLE I

\begin{tabular}{c|c|c}
\hline \hline Quantity & Element type & Other info \\
\hline & & $\rho=7500 \mathrm{~kg} / \mathrm{m}^{3}$, \\
plate & SOLID45 & $E=2.1 \times 10^{11} \mathrm{~Pa}$, \\
& & $v=0.3$ \\
piezo element & SOLID226 & Equivalent to PZT4, \\
& & $P=1600 \mathrm{~mm}^{2}$ \\
$V_{0}$ & - & $100 \mathrm{~V}$ \\
$V_{\mathrm{a} 1}-V_{\mathrm{a} 4}$ & - & $0.1-200 \mathrm{~V}$ \\
$\varphi_{1} \div \varphi_{4}$ & - & $0-360^{\circ}$
\end{tabular}

In Table I $V_{0}$ is voltage applied on square piezo element as plate excitation; $V_{1}-V_{4}$ are amplitudes of the voltages applied to actuators for vibration reduction; $\varphi_{1} \div \varphi_{4}$ are the phase angles of voltages applied to aforementioned actuators.

The goal of the analysis was to achieve as large vibration reduction, as possible. For this a goal function was created

$$
J=\min \left(\frac{\sum_{j=1}^{k} \frac{\sum_{i=1}^{n}\left|U_{j, i}\left(f_{i}\right)\right|}{n}}{k}\right),
$$

where $U_{j, i}$ - the voltage on sensor $j$ during $i$-th substep, $n$ - total number of substeps in analysis, $k$ - total number of sensors.

The optimization procedure for actuators voltage was done using internal ANSYS procedures with $J$ as objective function, and $V_{1}-V_{4}$ and $\varphi_{1} \div \varphi_{4}$ - design variables. The maximum number of iteration was set for 200 .

\section{Results}

The results of our analysis are shown in Table II.

From Table II it can be seen that when taking into account the shape of actuators for multiple modes reduction the square shape seems to be most effective, no matter whether it was a homogeneous or two-part type. If we compare the same results looking at piezoelements composition it is clear that homogeneous type seems to be less effective than two-part type.

Vibration level reduction for different cases.

TABLE II

\begin{tabular}{c|c|c|c|c|c|c}
\hline \hline \multirow{2}{*}{ Mode } & \multicolumn{2}{|c|}{ Square } & \multicolumn{2}{c|}{ Parallelogram } & \multicolumn{2}{c}{ Right angle triangle } \\
\cline { 2 - 7 } & homogeneous & two-part & homogeneous & two-part & homogeneous & two part \\
\hline 1 & 12.7 & 18.5 & 9.7 & 5.3 & 16.0 & 18.1 \\
2 or 3 & 22.8 & 18.2 & 21.9 & 23.9 & 26.1 & 30.5 \\
4 & 14.1 & 13.6 & 11.3 & 15.8 & 16.6 & 7.1 \\
5 & 28.5 & 26.8 & 26.8 & 32.0 & 27.4 & 34.7 \\
all & 10.8 & 10.9 & 7.6 & 8.7 & 4.4 & 5.6
\end{tabular}

For singular modes reduction and homogeneous type elements parallelograms were least effective for each mode, and square and triangle shaped elements being most effective for specific modes. Situation changes for two-part type elements, where the squares are least effective and triangles being the most (with the exception of mode $(2,2))$.

If looking at the results between different types of piezoelements it can be seen that for most cases homogeneous elements are less effective even though they have voltage applied to greater area (the biggest exception here are square shaped elements).

As shown in Fig. 2 when reducing several modes at once observed reduction corresponds rather with reduction of some of the modes and can amplify others. The high levels of voltage for 5th mode are the result of specific shape of the mode. Also during singular mode reduction it has been noticed that ANSYS inner optimization procedure sometimes gets stuck on local not global minimum.

\section{Conclusions}

Performed simulations allowed achieving significant vibration reduction with up to almost $35 \mathrm{~dB}$ when reducing singular modes and $11 \mathrm{~dB}$ when reducing multiple modes. This coupled with significant differences between actuators shapes and types used encourages physical studies.

Although "standard" shape of piezoelement was very efficient, this study showed that for some cases different shapes can be much more efficient. It should be noted 


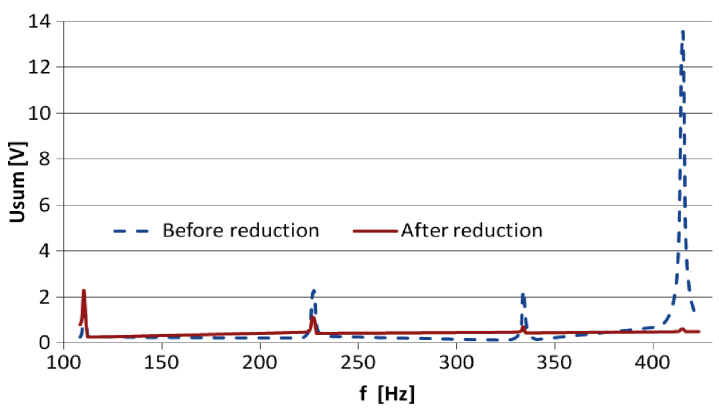

Fig. 2. Voltage on sensors before and after reduction of 4 modes simultaneously for square shaped homogeneous piezoelements.

that for presented "less standard" shapes there are still quite a few variables that could drastically change their efficiency, like parallelogram angles, different types of triangles, or the angles between elements and plate edges.

The reduction of actuator work area does not necessarily mean deterioration in its performance (in most cases it is quite the opposite). This shows quite a few possibilities such as using two-type piezoelements as both actuators and sensors, using the inner part as secondary actuator (with different voltage amplitude and phase values) for further improving its efficiency. Or simply changing actuator characteristics with applying the same voltage to whole element but with having its inner part from material with piezoconstants (which seems to be easiest to do in physical study).

Future works will aim to improve models, test additional shapes of piezoelements, adding acoustical volume (for noise reduction) performing physical tests and comparing their results with simulations.

\section{Acknowledgments}

This study is a part of the research project N N504078038 supported by the Ministry of Science and Higher Education, Poland.

\section{References}

[1] C.R. Fuller, S.J. Elliott, P.A. Nelson, Active Control of Vibration, Academic Press, London 1996.

[2] C.H. Hansen, S.D. Snyder, Active Control of Noise and Vibration, E\&FN Spon, London 1997.

[3] E. Dimitradis, C. Fuller, C. Rogers, J. Vibrat. Acoust. 113, 100 (1991).

[4] P. Gardonio, S.J. Elliott, J. Acoust. Soc. Am. 117, 2046 (2005).

[5] P. Gardonio, Sensor-Actuator Transducers for SmartPanels, ACTIVE, Sydney 2006.

[6] K. Pietrusiewicz, Control Eng. Poland 5, 20 (2007) (in Polish).

[7] R. Trojanowski, J. Wiciak, Acta Phys. Pol. A 118, 168 (2010).

[8] A. Brański, G. Lipiński, Acta Phys. Pol. A $\mathbf{1 1 9}$ A-936 (2011).

[9] A. Brański, M. Borkowski, S. Szela, Acta Phys. Pol. A 118, 17 (2010).

[10] J.D. Sprofera, R.H. Cabell, G.P. Gibbs, R.L. Clark, J. Acoust. Soc. Am. 122, 271 (2007).

[11] M.S. Kozien, J. Wiciak, Acta Phys. Pol. A 116, 348 (2009).

[12] A. Kundu, A. Berry, J. Acoust. Soc. Am. 129, 726 (2011).

[13] M.M. Zhang, L. Cheng, Y. Zhou, J. Acoust. Soc. Am. 126, 36 (2009).

[14] N. Tanaka, M. Tanaka, J. Acoust. Soc. Am. 127, 3526 (2010).

[15] R. Trojanowski, J. Wiciak, Acta Phys. Pol. A 121, A148 (2012). 